\title{
CONTROLE DA ESTABILIDADE DE EMULSÕES MULTIPLAS
}

\section{CONTROL OF THE STABILITY OF MULTIPLE EMULSIONS}

\author{
Felipe de Freitas NUNES'; Letícia da Mata LAZINSKI²; Cibéli MARTINS'; Gislene Mari \\ FUJIWARA ${ }^{3}$; Fernando Cesar Martins BETIM ${ }^{1}$; Camila Freitas de OLIVEIRA'; Lígia Moura \\ BURCI $^{*}$; Samanta GOLIN"; Marilis Dallarmi MIGUEL5; Deise Prehss MONTRUCCHIO5; \\ Josiane de Fátima Gaspari DIAS 5 ; Vitor Alberto KERBER ${ }^{5}$; Sandra Maria Warumby ZANIN ${ }^{5}$ \\ 1 - Mestre em Ciências Farmacêuticas pela Universidade Federal do Paraná; \\ 2 - Aluna do curso de Farmácia na Universidade Federal do Paraná; \\ 3 - Doutora em Ciências Farmacêuticas pela Universidade Federal do Paraná; \\ 4 - Mestre em Farmacologia pela Universidade Federal do Paraná. \\ 5 - Professor do curso de Farmácia e da Pós-Graduação em Ciências Farmacêuticas na Universidade Federal \\ do Paraná. \\ Autor para correspondência: ligia.burci@gmail.com
}

\section{RESUMO:}

Caracterizadas por serem um sistema carreador complexo contendo duas emulsões distintas que coexistem simultaneamente, as emulsões múltiplas apresentam muitas vantagens e aplicações, contudo manter a sua estabilidade apresenta-se ainda como um grande desafio. Foi realizada uma revisão da literatura existente além de uma análise sobre os principais fatores que interferem no controle da estabilidade da emulsão múltipla. Dentre os fatores destaca-se a presença de eletrólitos, a concentração do componente ativo osmótico, a influência dos aditivos estabilizantes, a natureza da fase oleosa, a propriedade dos filmes interfaciais e a relação de volume entre as fases, assim como o método de preparo. A observação desses fatores pode propiciar a elaboração e o uso das emulsões múltiplas de maneira proveitosa e propiciar a sua maior aplicabilidade em produtos farmacêuticos.

Palavras-chave: emulsões, estabilidade, eletrólitos

\section{ABSTRACT:}

Characterized as a complex carrier system containing two distinct emulsions coexisting simultaneously, multiple emulsions have many advantages and applications, yet maintaining their stability is still a major challenge. A review of the existing literature was performed in addition to an analysis of the main factors that interfere in the control of the stability of the multiple emulsion. Among the factors, the presence of electrolytes, the concentration of the active osmotic component, the influence of the stabilizing additives, the nature of the oil phase, the properties of the interfacial films and the volume ratio between the phases, as well as the preparation method. The observation of these factors can lead to the elaboration and the use of the multiple emulsions in a profitable way and to propitiate its greater applicability in pharmaceutical products.

Keywords: emulsions, stability, electrolytes

\section{INTRODUÇÃO}

Emulsões múltiplas são definidas como sistemas carreadores complexos, em que 
duas emulsões distintas água-em-óleo $(A / O)$ e óleo-em-água $(O / A)$ coexistem simultaneamente em um único sistema. Os tipos mais comuns são água-em-óleo-em-água $\left(A_{1} / O_{/} A_{2}\right)$ ou óleo-em-água-em-óleo $\left(\mathrm{O}_{1} / \mathrm{A} / \mathrm{O}_{2}\right)$. (PEREIRA; GARCIA-ROJAS, 2015; KUMAR; KUMAR; MAHADEVAN KUMAR, 2012; AKHTAR et al., 2010; SCHMIDTS et al., 2010; MORAIS, 2008; COLE; WHATELEY, 1997).

O método de obtenção mais utilizado é o processo de re-emulsificação em duas etapas, onde inicialmente se prepara a emulsão primária $\left(A_{1} / O\right)$, emulsionando-a posteriormente em água para formar a emulsão múltipla $\left(A_{1} / O / A_{2}\right)$. As fases aquosas interna e externa estão separadas por uma camada de óleo e, devido a baixa estabilidade termodinâmica, requerem pelo menos dois agentes emulsionantes, um com característica lipofílica (EHL baixo), como por exemplo o monooleato de sorbitano - Span $80^{\circledR}$, para formação da emulsão primária, e outro com característica hidrofílica (EHL alto), como por exemplo o polioxietileno (20) monooleato de sorbitano - Tween $80^{\circledR}$, para atingir a emulsificação secundária e formar a emulsão múltipla, estabilizando 0 sistema. (AGRAWAL; KULKARNI; SHARMA, 2015; PEREIRA; GARCIA-ROJAS, 2015; SCHMIDT et al., 2015; AMID; MIRHOSSEINI, 2014; KUMAR; KUMAR; MAHADEVAN KUMAR, 2012; AKHTAR et al., 2010; SCHMIDTS et al., 2010; SCHMIDTS et al., 2009; MORAIS, 2008; CARLOTTI et al., 2005; COLE; WHATELEY, 1997; COLE; WHATELEY, 1995; OMOTOSHO, 1990; FLORENCE; WHITEHILL, 1982).

A estrutura dos glóbulos múltiplos $A_{1} / O / A_{2}$ é dependente da natureza do emulsionante. Estudos têm demostrado que é possível preparar três tipos diferentes de emulsão múltipla $A_{1} / O / A_{2}$, a partir de três emulsões base compostas de água-miristato de isopropila-água, preparadas com três emulsionantes hidrofílicos distintos, Brij $30^{\circledR}$ (Polioxietileno lauril éter), Triton $\mathrm{X}-165^{\circledR}$ (Octilfenol etoxilado) e sistema Span $80^{\circledR}$ (Monooleato de sorbitano) e Tween $80^{\circledR}$ (Polioxietileno [20] monooleato de sorbitano), na proporção (3:1). Verificou-se um caráter significativamente diferente entre as emulsões. Foi evidenciado pequenos glóbulos múltiplos (diâmetro médio de 8,6 $\mu \mathrm{m}$ ) com apenas uma gota aquosa interna na emulsão contendo Brij $30^{\circledR}$. Já na emulsão contendo Triton $\mathrm{X}-165^{\circledR} \mathrm{o}$ sistema apresentou glóbulos múltiplos maiores (diâmetro médio de $19 \mu \mathrm{m}$ ), contendo pequenas, porém numerosas gotículas aquosas internas. E no caso da emulsão com sistema Span $80^{\circledR}$ : Tween $80^{\circledR}$, a mesma apresentou glóbulos múltiplos muito grandes (diâmetro médio de $25 \mu \mathrm{m}$ ), com um grande número de gotículas internas. Sendo assim foi possível determinar que a estrutura do glóbulo múltiplo provavelmente é dependente da segunda etapa de emulsificação. (FLORENCE; WHITEHILL, 1982; FLORENCE; 
WHITEHILL, 1981).

Devido à complexidade das emulsões múltiplas criou-se um sistema de nomenclatura para caracterizar as fases constituintes do sistema. Por exemplo, a emulsão $A_{1} / O / A_{2}$ pode ser descrita pela formação de duas interfaces, em que a fase aquosa $A_{1}$ é dispersa na fase oleosa $(O)$ formando a emulsão primária $A_{1} / O$. $A$ emulsão $A_{1} / O$ então é redispersa na fase aquosa externa $\left(A_{2}\right)$, formando a emulsão múltipla $A_{1} / O / A_{2}$. (FLORENCE; WHITEHILL, 1982).

Historicamente os primeiros relatos a respeito do desenvolvimento de emulsões múltiplas provêm de 1965, em que foi desenvolvida uma emulsão múltipla como um novo sistema de liberação de componentes ativos. (COLE; WHATELEY, 1997; HERBERT, 1965).

Por sua vez, Engel, Riggi e Fahrenbach (1968) investigaram o possível uso de emulsões múltiplas $A_{1} / O / A_{2}$ para facilitar a absorção gastrointestinal de biopolímeros solúveis em água, normalmente não absorvíveis. Shichiri e colaboradores (1975) demonstraram que ratos com diabetes induzida apresentaram redução significativa nos níveis de glicose na urina, com a administração de emulsão múltipla $A_{1} / O / A_{2}$ de insulina.

Atualmente, devido à estrutura e propriedades, as emulsões múltiplas são de grande interesse para diversas áreas de aplicação, podendo ser empregadas na indústria cosmética, farmacêutica e alimentícia, atuando na veiculação de componentes ativos com liberação controlada, bem como na encapsulação de substâncias para mascarar o sabor desagradável e proteger de processos de degradação. Suas aplicações farmacêuticas potenciais incluem a utilização em vacinas e mobilização de enzimas. Para uso cosmético, podem ser utilizadas na incorporação de materiais incompatíveis e na proteção de ativos por dispersão na fase interna, como por exemplo a vitamina $C$. Existem também relatos de utilizações não farmacêuticas das emulsões multiplas, na separação de hidrocarbonetos. (SCHMIDT et al., 2015; MAHMOOD et al., 2013; HERNÁNDEZ-MARÍN; LOBATOCALLEROS; VERNON-CARTER, 2013; SIGWARD et al., 2013; KUMAR; KUMAR; MAHADEVAN, 2012; MURILLO-MARTÍNEZ et al., 2011; AKHTAR et al., 2010; LOBATOCALLEROS et al., 2008; COLE; WHATELEY, 1997; COLE; WHATELEY, 1995; OMOTOSHO, 1990).

Assim, as emulsões múltiplas apresentam muitas vantagens e aplicações, porém, obter formulações estáveis ainda é um desafio. A necessidade de utilização de grandes quantidades de emulsionantes dificulta a formulação de emulsões em grau farmacêutico, 
motivo pelo qual existem poucas emulsões múltiplas farmacêuticas no mercado. (MAHMOOD; AKHTAR; MANICKAM, 2014; SCHMIDTS et al, 2009).

\section{METODOLOGIA}

Trabalho exploratório que objetivou elencar etapas e fatores importantes no controle da estabilidade de uma emulsão múltipla. Para isso foi realizada uma revisão fichas técnicas e em sites de busca no Brasil, para essa verificação.

\section{REVISÃO DA LITERATURA}

\subsection{Controle da estabilidade de emulsões múltiplas $A_{1} / O / A_{2}$}

As emulsões múltiplas água-em-óleo-em-água $\left(\mathrm{A}_{1} / \mathrm{O} / \mathrm{A}_{2}\right)$ devido à complexidade de sua estrutura não são sistemas estáveis. Assim, há a necessidade de avaliar os fatores que causam a desestabilização para que se possa preparar estes produtos com estabilidade adequada para possível comercialização. (KUMAR; KUMAR; MAHADEVAN, 2012; CARLOTTI et al., 2005; FLORENCE; WHITEHILL, 1982).

A instabilidade destes sistemas emulsionados múltiplos pode ocorrer pelos mecanismos de (i) coalescência das gotículas de água na fase $A_{1} / O$ (AKHTAR et al., 2010; FLORENCE; WHITEHILL, 1981); (ii) coalescência dos glóbulos de óleo na emulsão $A_{1} / O / A_{2}$ (AKHTAR et al., 2010; FLORENCE; WHITEHILL, 1981); (iii) ruptura do filme oleoso entre as fases aquosas interna e externa, resultando na perda das gotículas aquosas internas (AKHTAR et al., 2010; SCHMIDTS et al., 2009; FLORENCE; WHITEHILL, 1981); (iv) passagem da água bem como de fármacos, ativos ou aditivos solúveis na água através da camada de óleo entre as fases aquosas interna e externa do sistema múltiplo. (SCHMIDTS et al. 2009; FLORENCE; WHITEHILL, 1981).

Nessa revisão incluiremos os fatores específicos para o controle da estabilidade de emulsões múltiplas $A_{1} / O / A_{2}$.

\subsubsection{Presença de eletrólitos}

A presença de eletrólitos parece ser um dos mais importantes fatores que determinam a estabilidade e a liberação de componentes ativos ou aditivos das gotículas 
internas das emulsões múltiplas $\mathrm{A}_{1} / \mathrm{O} / \mathrm{A}_{2}$. Isto ocorre através do balanço da pressão osmótica entre as fases aquosas interna $\left(A_{1}\right)$ e externa $\left(A_{2}\right)$ ou, pela formação de uma camada interfacial rígida entre a fase oleosa e a fase aquosa interna $\left(A_{1}\right)$ do sistema múltiplo (AKHTAR et al., 2010; FLORENCE; WHITEHILL, 1982).

Os efeitos dos eletrólitos acontecem de duas maneiras: (i) osmótico, peculiar aos sistemas múltiplos e (ii) interfacial (FLORENCE; WHITEHILL, 1982).

Considerando os efeitos osmóticos que podem ocorrer nos sistemas múltiplos considera-se que as moléculas de água tanto quanto componentes solúveis na água possam passar de uma fase aquosa para a outra através da fase oleosa que atua como uma membrana semipermeável que é muitas vezes referida como fase de membrana ou fase membrana (FLORENCE; WHITEHILL, 1982).

Se a pressão osmótica é maior na fase aquosa interna $\left(A_{1}\right)$, a água pode passar para esta fase resultando em dilatação das gotículas internas que eventualmente rompem, liberando o conteúdo. O reverso é verdadeiro se a pressão osmótica é maior na fase aquosa externa $\left(A_{2}\right)$, resultando em transferência de água da fase aquosa interna para a externa causando contração das gotículas internas (FLORENCE; WHITEHILL, 1982).

Se a diferença de pressão osmótica através da camada oleosa é extrema, então a passagem de água é tão rápida que quase imediatamente ocorre ruptura dos glóbulos de óleo com expulsão das gotículas internas. Este fato ocorre com frequência onde a camada de óleo é fina. Outras substâncias que não eletrólitos, como proteínas, açúcares e fármacos podem também exercer este efeito (AKHTAR et al., 2010; SCHMIDTS et al., 2010; FLORENCE; WHITEHILL, 1982).

Quando a camada de óleo rompe, a fase aquosa interna em gotícula desaparece instantaneamente, seguido pela mistura da fase aquosa interna com o meio aquoso externo e permanecendo no sistema um simples glóbulo de óleo (FLORENCE; WHITEHILL, 1982).

Em relação aos efeitos interfaciais foi observado que fatores outros que gradientes osmóticos afetam a passagem do fármaco. Foi sugerido que o cloreto de sódio ( $\mathrm{NaCl}$ ) compete com o emulsionante pelas moléculas de água na interface interna $A_{1} / O$, o que resultaria em uma camada interfacial rígida que poderia atuar como uma barreira mecânica mais efetiva para a transferência do fármaco (FLORENCE; WHITEHILL, 1982).

Em trabalho de Schmidts e colaboradores (2010) foi constatado que as diferenças entre emulsões contendo $\mathrm{NaCl}$ e sulfato de magnésio $\left(\mathrm{MgSO}_{4}\right)$ são devidas a diferentes interações dos íons $\mathrm{Na}^{+}$e $\mathrm{Mg}^{2+}$ com os emulsionantes. Observou-se que os eletrólitos influenciam as propriedades dos emulsionantes pelo efeito da solubilização por salificação 
(salting in) ou pela diminuição da solubilização por salificação (salting out). Os cátions bivalentes, tais como $\circ \mathrm{Mg}^{2+}$, salt-in os emulsionantes não iônicos polietoxilados por complexação dos cátions com o átomo de oxigênio do éter. Por outro lado, os cátions monovalentes como o $\mathrm{Na}^{+}$salt-out os emulsionantes pois competem pela água de hidratação na parte polar do emulsionante, isto é, promovem a desidratação das cadeias de polioxietileno, diminuindo a sua solubilidade, o que desestabiliza energeticamente a emulsão.

Em emulsões contendo Steareth- $20^{\circledR}$ como emulsionante, as propriedades de salting-in dos íons magnésio mostraram um efeito positivo na estabilidade da emulsão em comparação a outros aditivos por um período de 6 meses de armazenamento. Os sistemas mostraram estabilidade adequada pois não ocorreu nem separação nem inversão de fases. A utilização do $\mathrm{MgSO}_{4}$ na fase aquosa interna forneceu viscosidade estável aos sistemas emulsionados ao longo do tempo (SCHMIDTS et al., 2010).

\subsubsection{Concentração do componente ativo osmótico}

A estabilização depende da concentração escolhida do componente ativo osmótico, previamente acrescentado à fase aquosa interna do sistema. Existem diversos fatores que podem influenciar a migração dos componentes ativos osmóticos, como o coeficiente de partição, ionização, densidade de carga, peso molecular e mobilidade molecular. Para se obter uma formulação estável, a concentração de eletrólitos tem que ser alta o suficiente para regular a pressão de Laplace mas ao mesmo tempo suficientemente baixa para evitar os efeitos osmóticos. Assim, o balanço da pressão osmótica pode ser explicado pela neutralização da pressão de Laplace que está associada à curvatura da superfície do glóbulo. Isto resulta em decréscimo no mecanismo de maturação de Ostwald (Ostwald ripening) nas emulsões, que são usualmente polidispersas. Os glóbulos menores terão solubilidade maior quando comparados aos maiores, devido a maior curvatura interfacial. Com o tempo, os glóbulos menores tendem a desaparecer e suas moléculas difundem para a fase dispersante depositando nos glóbulos maiores e acarretando variação na distribuição de tamanho a valores maiores. (KUMAR; KUMAR; MAHADEVAN, 2012; SCHMIDTS et al., 2010; MORAIS, 2008).

Existem diversos mecanismos possíveis por meio dos quais as substâncias podem ser transferidas através da camada oleosa em um sistema $A_{1} / O / A_{2}$. Dois mecanismos são sugeridos para a possível permeação de água e ativos ionizados através da fase oleosa 
nestes sistemas emulsionados: (i) por transporte em micelas mistas e inversas de emulsionantes hidrofóbicos e hidrofílicos e (ii) as moléculas de água podem difundir através de lamelas muito finas de emulsionantes formadas onde a camada oleosa é muito fina. Foi sugerido que estes mecanismos provavelmente ocorrem quando existe uma diferença de pressão osmótica entre as duas fases aquosas. (SCHMIDTS et al., 2009; FLORENCE; WHITEHILL, 1982; FLORENCE; WHITEHILL, 1981).

Para substâncias não ionizadas solúveis em lipídios a difusão no óleo parece ser o mecanismo mais importante de transporte em sistemas múltiplos. Este mecanismo é dependente da natureza da substância, incluindo sua constante de dissociação, a natureza do óleo e o pH da fase aquosa. (AKHTAR et al, 2010; FLORENCE; WHITEHILL, 1982; FLORENCE; WHITEHILL, 1981).

\subsubsection{Influência de aditivos estabilizantes}

O maior problema associado às emulsões múltiplas $A_{1} / O / A_{2}$ é a cremação, mecanismo que ocorre provavelmente devido ao tamanho grande das gotículas múltiplas. Pode-se reduzir a cremação pelo aumento da concentração do emulsionante secundário no sistema, contudo, pode acarretar toxicidade e diminuição na liberação do ativo. (FLORENCE; WHITEHILL, 1982).

Alguns aditivos estabilizantes podem ser acrescentados às emulsões múltiplas para aumentar a sua estabilidade. Estes componentes incluem os agentes gelificantes ou de aumento de viscosidade que são acrescentados às fases aquosas interna ou externa do sistema e incluem gelatina, metilcelulose e agentes espessantes similares. (KUMAR; KUMAR; MAHADEVAN, 2012).

Um produto bastante utilizado como aditivo espessante é a goma xantana, um polissacarídeo de alto peso molecular (200.000 Da). Contêm D-glucose, D-manose e ácido glucurônico em sua estrutura química e é classificada como sendo um biopolímero de cadeia ramificada e aniônica. Dentre as propriedades atua como espessante e estabilizante. Possui elevada viscosidade em baixas concentrações. Soluções aquosas são estáveis em ampla faixa de $\mathrm{pH}(\mathrm{pH} 3-12)$, com estabilidade máxima em $\mathrm{pH} 4-10$ e temperatura de $10-60^{\circ} \mathrm{C}$. Suas soluções são também estáveis na presença de enzimas, sais, ácidos e bases. A goma xantana, por ser um material aniônico não é normalmente compatível com emulsionantes catiônicos, polímeros, ou conservantes, quando ocorre precipitação. Emulsionantes aniônicos e anfotéricos em concentração acima de 15\% (m/v) 
causam precipitação da goma xantana em solução (SCARIOTTO, 2013; ROWE; SHESKEY; QUINN, 2009; TONELI; MURR; PARK, 2005). Possui compatibilidade e estabilidade com a maioria dos sais metálicos. O caráter iônico nas moléculas de goma xantana aumenta a sua hidratação. (AMID; MIRHOSSEINI, 2014).

O efeito da incorporação de aditivos espessantes na fase aquosa interna da emulsão pode ser atribuído a formação de um filme polimérico rígido ou complexo macromolecular através das interfaces óleo/água, ou seja, através de interação interfacial entre macromoléculas na fase aquosa interna e o emulsionante lipofílico não iônico na fase oleosa do sistema. Este filme atua protegendo os glóbulos da coalescência e aumentando a estabilidade do sistema a longo prazo. (KUMAR; KUMAR; MAHADEVAN, 2012; SCHMIDTS et al., 2010; OMOTOSHO, 1990).

O uso de um agente espessante como o álcool polivinílico na fase aquosa externa pode reduzir a cremação, mas deve-se ficar atento que a emulsão retenha suas características reológicas (FLORENCE; WHITEHILL, 1982). Também podem ser utilizados agentes complexantes que conduzem à formação de fases cristalinas líquidas na interface $\mathrm{O} / \mathrm{A}_{2}$, como o álcool cetílico e, agentes gelificantes para a fase oleosa como 0 monoestearato de alumínio. (KUMAR; KUMAR; MAHADEVAN, 2012; FLORENCE; WHITEHILL, 1982).

Trabalhos prévios de Omotosho e colaboradores (1986) relataram a estabilização de emulsões múltiplas $A_{1} / O / A_{2}$ através de interação interfacial entre albumina sérica bovina na fase aquosa interna $A_{1}$ e monooleato de sorbitano (Span $80^{\circledR}$ ) na fase oleosa. Pode-se observar o complexo interfacial pela formação de um filme visível na interface óleo/água e pelo aumento da estabilidade do sistema múltiplo contendo como aditivo osmoticamente ativo o cloreto de sódio $(\mathrm{NaCl})$.

Em outro trabalho de Omotosho (1990) foram avaliadas emulsões múltiplas contendo fosfato de cloroquina na fase interna do sistema estabilizado por interação interfacial entre monooleato de sorbitano $\left(\right.$ Span $80^{\circledR}$ ) e as macromoléculas acácia, gelatina e polivinilpirrolidona. Os glóbulos de óleo múltiplos variaram em tamanho dependendo de qual macromolécula estava presente na fase interna, com diâmetro médio de 27,3 $\mu$ m para a polivinilpirrolidona; 36,7 $\mu \mathrm{m}$ para a acácia; 44,8 $\mu \mathrm{m}$ para a gelatina. A despeito de diferenças entre as macromoléculas na estabilidade da emulsão, estes sistemas forneceram emulsões mais estáveis do que as preparadas com Span $80^{\circledR}$ livre como emulsionante primário. Quanto à liberação do fosfato de cloroquina as diferenças observadas podem ser devidas ao tamanho da gotícula da fase aquosa interna e da força 
mecânica do filme interfacial na interface $A_{1} / O$. As emulsões múltiplas armazenadas por duas semanas mostraram um índice de liberação mais baixo de fosfato cloroquina quando comparadas àquelas recém preparadas. Pode-se atribuir a dois fatores estes resultados. Primeiro, que o filme interfacial resultante da interação entre Span $80^{\circledR}$ e a macromolécula torna-se mais rígido com o tempo, assim formando uma barreira mais eficiente contra o transporte do fosfato de cloroquina encapsulado. Segundo, a coalescência entre as gotículas, que poderia conduzir a um aumento no tamanho do glóbulo, redução da área interfacial disponível para o transporte do fármaco e redução no índice de liberação do fosfato de cloroquina. Contudo, não ocorreu aumento significativo no tamanho médio dos glóbulos, sugerindo que o fosfato de cloroquina é liberado do sistema por processo de difusão através da membrana líquida oleosa e não por quebra da emulsão.

Em trabalho de Schmidts e colaboradores (2010) dois diferentes derivados da celulose, a hidroxietilcelulose e a sódio-carboximetilcelulose, foram incorporados na fase aquosa interna do sistema múltiplo $\mathrm{A}_{1} / \mathrm{O} / \mathrm{A}_{2}$. A maioria dos derivados da celulose dissolve em água fria e são principalmente usados para o controle da viscosidade por gelificação. Como resultado, a adição do espessante na fase aquosa interna não afetou as propriedades físicas da emulsão primária $A_{1} / O$ subsequente à produção. Utilizando diferentes emulsionantes não iônicos polietoxilados observou-se diferenças nas interações entre os derivados da celulose e os grupos químicos dos emulsionantes, como grupos éster e éter, possibilitando ou não a formação do sistema múltiplo. A adição destes derivados da celulose resultou em decréscimo na separação de fases nas formulações testadas, mas pouco interferiu nas propriedades físico-químicas das emulsões múltiplas e nem na liberação dos eletrólitos encapsulados.

Em trabalho de Cole e Whateley (1995) foram desenvolvidos complexos entre emulsionantes hidrofílicos Pluronic $^{\circledR}$ (Poloxamer ${ }^{\circledR}$ ) e ácido poli-acrílico \{(PAA), Carbopol $\left.907^{\circledR}\right\}$ que possam interagir com o emulsionante lipofílico e aumentar a estabilidade de emulsões múltiplas $A_{1} / O / A_{2}$. As emulsões foram preparadas pelo processo em duas etapas e a fase aquosa de cada emulsão primária $A_{1} / O$ continha solução do complexo Pluronic ${ }^{\circledR}: P A A$, que reúne as propriedades de um polieletrólito (PAA) e um emulsionante hidrofílico polimérico (Pluronic ${ }^{\circledR}$ ) na mesma molécula. A estabilidade das emulsões preparadas com estes complexos, que possuem propriedades bioadesivas, possui relação aos tamanhos das partículas dos complexos Pluronic $^{\circledR}:$ PAA e do tipo de emulsionante lipofílico utilizado na fase oleosa. 
Quando este complexo está em contato direto com uma fase oleosa contendo um emulsionante lipofílico polimérico como o Pluronic $L 101{ }^{\circledR}$, então o aumento na estabilidade pode ser devido a interação polímero-polímero na interface. Se a fase oleosa contém um emulsionante lipofílico de peso molecular pequeno como o Span $80^{\circledR}$, a estabilidade pode ser devida a um aumento na adsorção na interface no sistema.

O propósito da estabilização da interface $A_{1} / O$ na preparação de emulsões múltiplas é o de reduzir o potencial para a coalescência das gotículas de água encapsuladas ou a sua expulsão dos glóbulos de óleo múltiplos ou, para prevenir a coalescência dos glóbulos de óleo múltiplos.

\subsubsection{Natureza da fase oleosa}

As propriedades da fase oleosa são importantes na determinação da estabilidade do sistema e controle dos índices de transferência dos solutos. A natureza da fase oleosa pode afetar, acentuadamente, as propriedades do sistema, influenciando no tamanho das gotículas aquosas internas e dos glóbulos múltiplos (COLE; WHATELEY,1995). Destas propriedades, a mais importante é a viscosidade. (FLORENCE; WHITEHILL, 1982).

A maioria dos óleos formam emulsões múltiplas se as condições de processamento adequadas forem aplicadas. (FLORENCE; WHITEHILL, 1982).

Estudos foram conduzidos em diferentes sistemas $A_{1} / O / A_{2}$ usando uma mistura de dois óleos: um isoparafínico de alta viscosidade e um parafínico leve. As proporções relativas de cada óleo afetaram a viscosidade da fase oleosa. Quanto mais alta a concentração de óleo de alta viscosidade maior a estabilidade do sistema em relação à ruptura do filme oleoso e o controle dos índices de transferência dos solutos através da membrana oleosa. (FLORENCE; WHITEHILL, 1982).

Para produtos farmacêuticos, os óleos utilizados incluem os hidrocarbonetos refinados como parafina líquida leve, esqualeno e ésteres de ácidos graxos de cadeia longa incluindo os óleos vegetais, por exemplo oleato de etila e miristato de isopropila. (KUMAR; KUMAR; MAHADEVAN, 2012; FLORENCE; WHITEHILL, 1982).

Outros óleos utilizados incluem os vários óleos de origem vegetal como o óleo de oliva, sésamo, soja, amendoim e girassol se purificados corretamente, pois a fase oleosa a ser empregada em uma emulsão farmacêutica deve ser não tóxica. (KUMAR; KUMAR; MAHADEVAN, 2012; FLORENCE; WHITEHILL, 1982).

Como regra geral, os óleos minerais produzem emulsões múltiplas mais estáveis 
que aquelas produzidas com óleos vegetais. A ordem decrescente de estabilidade e percentagem de encapsulação tem sido apontada como sendo parafina líquida leve > esqualeno > óleo de sésamo > óleo de amendoim. (KUMAR, KUMAR; MAHADEVAN, 2012).

Dentre os óleos minerais, aqueles de viscosidade elevada são os que produzem as emulsões mais estáveis. A elevada viscosidade dificulta ou impede a difusão de água e de substâncias solúveis na água entre as fases aquosas interna e externa do sistema múltiplo e as alterações na emulsão ocorrem significativamente mais devagar. (KUMAR; KUMAR; MAHADEVAN, 2012; JIGAR et al., 2011; FLORENCE; WHITEHILL, 1982).

\subsubsection{Propriedades dos filmes interfaciais}

Um emulsionante lipofílico e outro hidrofílico são necessários para formar uma emulsão estável $A_{1} / O / A_{2}$. Assim, tanto a composição química quanto a concentração utilizada dos emulsionantes na obtenção destes sistemas múltiplos tem sido estudados por diversos autores. (KUMAR; KUMAR; MAHADEVAN, 2012; SCHMIDTS et al., 2009; CARLOTTI et al., 2005; GEIGER et al.,1998; COLE; WHATELEY, 1995).

Emulsionantes com propriedades químicas compatíveis com os componentes da emulsão e com valores de EHL adequados ao sistema são necessários para a obtenção de emulsões estáveis. Os valores de EHL do emulsionantes hidrofílicos usados no preparo de emulsões múltiplas $A_{1} / O / A_{2}$ variam de 12 a 22 e são quimicamente ésteres e éteres de ácidos graxos polietoxilados ou poliméricos. Para estabilizar a interface da emulsão primária $A_{1} / O$, o valor de $E H L$ do emulsionante deve ficar entre 2 e 7. (KUMAR; KUMAR; MAHADEVAN, 2012; FLORENCE; WHITEHILL, 1982).

Em estudos realizados por Schmidts e colaboradores (2009) foram utilizados vários emulsionantes hidrofílicos como ésteres de sorbitano polietoxilado e ácidos graxos, conhecidos como polisorbatos ou Tweens ${ }^{\circledR}$; ésteres de derivados polietoxilados de ácido esteárico, como exemplo o PEG - 20 stearate ${ }^{\circledR}$ e ésteres da sucrose, como a Sucrose palmitate ${ }^{\circledR}$. Também foram utilizados éteres de álcoois graxos polietoxilados, como exemplo o Steareth $-20^{\circledR}$ e o emulsionante polimérico Poloxamer $407^{\circledR}$. Neste estudo foi possível observar a influência do valor de EHL do sistema como também a composição química e compatibilidade do emulsionante hidrofílico influenciam as propriedades e a estabilidade das emulsões múltiplas $A_{1} / O / A_{2}$. Foi demonstrado que emulsões múltiplas com ótimas propriedades e estabilidade foram obtidas usando álcoois graxos polietoxilados. Estes PEG éteres possuem propriedades semelhantes aos PEG ésteres, mas possuem maior 
estabilidade hidrolítica, permitindo seu uso em valores extremos de pH. Também são capazes de tolerar altos níveis de eletrólitos quando comparados aos emulsionantes do tipo éster devido a ausência de ligação éster.

Este estudo também demonstrou que o tamanho dos glóbulos e a encapsulação de $\mathrm{NaCl}$ foram dependentes do valor de EHL mas não a viscosidade do sistema. Um EHL entre 14 e 15 forneceu os menores glóbulos e a melhor encapsulação, o aumento do EHL aumentou o tamanho dos glóbulos e a liberação do $\mathrm{NaCl}$ para a fase aquosa externa do sistema. Também foi observado que uma mistura de emulsionantes ao invés de um único emulsionante com o mesmo valor de EHL, coopera na obtenção de glóbulos menores.

A concentração dos emulsionantes também afeta a estabilidade dos sistemas emulsionados. Quantidades muito pequenas podem resultar em sistemas instáveis e muito elevadas podem ser tóxicas e mesmo desestabilizantes do sistema. (KUMAR; KUMAR; MAHADEVAN, 2012).

Contudo, para cada sistema emulsionado existe uma concentração adequada de emulsionantes para sua estabilização. Uma concentração baixa pode ocasionar degradação rápida da emulsão e uma alta pode aumentar a sua viscosidade, porém há o inconveniente da toxicidade. De maneira geral usa-se de 1 a $10 \%$ de emulsionante (KUMAR; KUMAR; MAHADEVAN, 2012; FLORENCE; WHITEHILL, 1982).

Deve-se considerar um índice de concentração 10 vezes maior de emulsionante lipofílico para a fase $A_{1} / O$ em relação à fase $O / A_{2}$ devido à solubilização das moléculas do emulsionante primário lipofílico na fase aquosa externa $\mathrm{O} / \mathrm{A}_{2}$. Esta solubilização acontece quando a concentração do emulsionante secundário hidrofílico excede a concentração micelar crítica (CMC). (FLORENCE; WHITEHILL, 1982).

Se ocorre aumento de concentração do emulsionante secundário hidrofílico, mais do emulsionante primário lipofílico pode ser incorporado nas micelas, ocasionando a diminuição de sua concentração na fase oleosa e o sistema sofre desestabilização, que pode conduzir à ruptura da camada oleosa, resultando na perda das gotículas aquosas internas. (FLORENCE; WHITEHILL, 1982).

Contudo, deve-se levar em consideração que nas emulsões múltiplas $A_{1} / O / A_{2} 0$ emulsionante hidrofílico pode sofrer a influência da migração do emulsificante lipofílico utilizado para o preparo da primeira emulsão $A_{1} / O$. Assim, o valor do $E H L$ na interface $O / A_{2}$ não corresponde mais ao valor do EHL do emulsionante hidrofílico utilizado mas sim à soma de ambos, incluso as concentrações de cada um no sistema (do inglês "weighted HLB"). Este fato relaciona-se à inversão da emulsão múltipla $A_{1} / O / A_{2}$ em $O / A$ e ocorre quando o 
EHL correspondente à soma dos emulsificantes presentes no sistema for maior que 10 . (SCHMIDTS et al., 2009; FRENKEL; SHWARTZ; GARTI, 1983).

O emulsionante lipofílico, durante a dilatação de fase que acontece quando ocorre fluxo de água da fase aquosa externa para a fase aquosa interna do sistema, pode difundir da primeira para a segunda interface, conferindo rigidez à membrana, ou da fase oleosa para a primeira interface, resultando no decréscimo da coalescência das gotículas aquosas durante a dilatação. A concentração do emulsionante lipofílico desempenha um papel predominante neste mecanismo. Parece que de um lado, a capacidade de dilatação do glóbulo aumenta com a sua concentração e, por outro, quanto maior a dilatação do glóbulo de óleo, menor a liberação de fármaco hidrossolúvel. (KUMAR; KUMAR; MAHADEVAN, 2012; GEIGER et al., 1998; JAGER-LEZER et al. 1997).

O Tween $80^{\circledR}$ é frequentemente utilizado em combinação com o Span $80^{\circledR} \mathrm{em}$ emulsões múltiplas $A_{1} / O / A_{2}$ devido à estrutura química semelhante de ambos. Tem sido observado que, na maioria dos casos, as emulsões mais estáveis são formadas quando os emulsionantes possuem o mesmo comprimento de cadeia hidrocarbonada. (SCHMIDTS et al., 2009).

\subsubsection{Relação de volume entre as fases, método de preparo e variáveis de processo}

Em trabalho desenvolvido por Matsumoto, Kita e Yonezawa (1976) foi constatado que o volume da fase interna $A_{1} / O$ não apresentava efeito significativo na obtenção da emulsão $A_{1} / O / A_{2}$ sob as condições experimentais estudadas. Este estudo demonstrou que as emulsões $A_{1} / O / A_{2}$ podem ser preparadas utilizando ampla variação de volume na fase interna $A_{1} / O$, em intervalo ótimo de 25-50\%. (FLORENCE; WHITEHILL, 1982).

Resultados interessantes obtidos por Matsumoto, Kita e Yonezawa (1976) sugeriram que $o$ volume da fase aquosa secundária $A_{1} / O / A_{2}$ influencia na formação dos glóbulos múltiplos sob um intervalo de frações de baixo volume. Quando o volume $A_{1} / O / A_{2}$ ultrapassou cerca de 0,4 não ocorreram efeitos significativos. Foi também observado que o volume da fase interna $A_{1} / O$ influenciou a liberação de substâncias encapsuladas na fase aquosa interna. (FLORENCE; WHITEHILL, 1982).

Em trabalho de Florence e Whitehill (1982) os autores sugerem que uma típica emulsão $A_{1} / O / A_{2}$ deve ser preparada da seguinte maneira: (i) a emulsão primária $A_{1} / O$ pode ser formulada com miristato de isopropila $(47,5 \%)$, emulsionante lipofílico monooleato de sorbitano (Span $\left.80^{\circledR}\right)(2,5 \%)$ e água destilada q.s.p. $100 \%$. (ii) A emulsão múltipla água-em- 
óleo-em-água $\left(A_{1} / O / A_{2}\right)$ pode então ser preparada adicionando a emulsão primária $A_{1} / O$ (50\%) em uma solução aquosa contendo o emulsionante hidrofílico polioxietileno (20) monooleato de sorbitano (Tween $\left.80^{\circledR}\right)(2 \%)$.

Em trabalho de Florence e Whitehill (1981) emulsões múltiplas foram preparadas por processo em duas etapas da seguinte maneira: (i) a fase aquosa $A_{1}$ foi emulsionada em igual quantidade de óleo contendo $5 \% \mathrm{~m} / \mathrm{m}$ de Span $80^{\circledR}$ por meio de um pequeno misturador por vibração para formar a emulsão primária $A_{1} / O$. (ii) $A$ emulsão $A_{1} / O$ foi reemulsionada da mesma maneira em igual quantidade de água contendo $2 \% \mathrm{~m} / \mathrm{m}$ de emulsionante ou sistema emulsionante hidrofílico.

Em trabalho de Jigar e colaboradores (2011) emulsões múltiplas contendo o fármaco atorvastatina foram preparadas por processo de emulsificação em duas etapas: (i) preparo da emulsão primária e (ii) emulsificação secundária. Considerando o volume entre as fases, na emulsificação primária $12,0 \mathrm{~mL}$ de água destilada foram acrescentadas a 28,0 $\mathrm{mL}$ de fase oleosa contendo o emulsificante primário. Na emulsificação secundária $30,0 \mathrm{~mL}$ da emulsão primária viscosa foi posteriormente emulsificada com uma fase aquosa externa contendo o emulsificante secundário. Diferentes variáveis foram analisadas como o tipo e concentração dos emulsionantes, velocidade e tempo de rotação e fração de volume das fases interna e externa do sistema.

Dos resultados obtidos para a emulsão primária o emulsionante Span $60^{\circledR} \mathrm{em}$ concentração de $10 \%$ forneceu boa estabilidade ao sistema e alta eficiência de encapsulação em velocidade de $5000 \mathrm{r} / \mathrm{min}$ por tempo de 10 minutos e fração de volume de fase interna: fase externa (30:70). Esta emulsão primária foi utilizada para a emulsificação secundária utilizando Tween $80^{\circledR}$ em concentração de $16 \%$ para boa estabilidade e máxima capacidade de encapsulação em velocidade de 1500 r/min por tempo de 7 minutos e mantido a fração de volume de fase interna : fase externa (30:70).

Em trabalho desenvolvido por Cole e Whateley (1995) onde foram utilizados complexos de emulsionante hidrofílico polimérico e polímero de ácido poli-acrílico na fase aquosa interna do sistema 0 índice de relação de fases $A_{1} / O / A_{2}$ foi de 1:2:3, respectivamente.

A quantidade de água dispersada na emulsão primária $A_{1} / O$, expressa como um índice de volume de fases $A_{1} / O / A_{2}$, pode influenciar tanto a obtenção quanto a estabilidade do sistema emulsionado final (KUMAR; KUMAR; MAHADEVAN, 2012). 


\section{REFERÊNCIAS}

AGRAWAL, A.; KULKARNI, S.; SHARMA, S.B. Recent advancements and applications of multiple emulsions. International Journal of Advances in Pharmaceutics, ISSN: 2320 4923, Vol. 4 Issue 6, p.94-103, 2015.

AKHTAR, N.; AHMAD, M.; KHAN, H.M.; GULFISHAN, A.J.; MAHMOOD, A.; UZAIR, M. Formulation and characterization of a multiple emulsion containing $1 \%$ I-ascorbic acid. Bulletin of the Chemical Society of Ethiopia.,v.24(1): p.1-10, 2010.

AMID, B. T.; MIRHOSSEINI, H. Stabilization of water in oil in water (W/O/W) emulsion using whey protein isolate-conjugated durian seed gum: Enhancement of interfacial activity through conjugation process. Colloids and surfaces B: Biointerfaces, v.113, p.107-114. 2014.

CARLOTTI, M.E.; GALLARATE, M.; SAPINO, S.; UGAZIO, E. W/O/W Multiple Emulsions for Dermatological and Cosmetic Use, Obtained with Ethylene Oxide Free Emulsifiers. Journal of Dispersion Science and Technology, v.26(2), p.183-192, 2005.

COLE, M.L.; WHATELEY, T.L. Preparation of Stable Multiple W/O/W Emulsions Using Pluronic (Polaxamer): Poly(acrylic Acid) Complexes. Journal of Colloid and Interface Science, v.175 (2): p.281-288, 1995.

COLE, M.L.; WHATELEY, T.L. Release rate profiles of theophylline and insulin from stable multiple w/o/w emulsions. Journal of Controlled Release, v.49: p.51-58, 1997.

ENGEL, R.H.; RIGGI, S.J.; FAHRENBACH, M.J. Insulin-intestinal absorption as water-inoil-in-water emulsions, Nature, v.219, p.856-857, 1968.

FLORENCE, A.T.; WHITEHILL, D. Some features of breakdown in water-in-oil-in-water multiple emulsions. Journal of Colloid and Interface Science, v.79 (1), p.243-256, 1981.

FLORENCE, A.T.; WHITEHILL, D. The formulation and stability of multiple emulsions. Review Article. International Journal of Pharmaceutics, v.11, p.277-308, 1982. 
FRENKEL, M.; SHWARTZ, R.; GARTI, N. Multiple emulsions: I. Stability: Inversion, apparent and weighted HLB. Journal of Colloid and Interface Science, v.94(1), p.174-178, 1983.

GEIGER, S.; TOKGOZ, S.; FRUCTUS, A.; JAGER-LEZER, N.; SEILLER, M.; LACOMBE, C.; GROSSIORD, J. L. Kinetics of swelling-breakdown of W/O/W multiple emulsion: possible mechanisms for the lipophilic surfactant effect. Journal of Controlled Release, v.52, p.99$107,1998$.

HERBERT, W.J. Multiple emulsions, a new form of mineral adjuvant, Lancet, v.11 - 771, 1965.

HERNÁNDEZ-MARÍN, N.Y.; LOBATO-CALLEROS, C.; VERNON-CARTER, E.J. Stability and rheology of water-in-oil-in-water multiple emulsions made with protein-polysaccharide soluble complexes. Journal of Food Engineering, v.119, p.181-187, 2013.

JAGER- LEZER, N.; TERRISSE, I.; BRUNEAU, F.; TOKGOZ, S.; FERREIRA, L.; CLAUSSE, D.; SEILLER, M.; GROSSIORD, J. L. Influence of lipophilic surfactant on the release kinetics of hydrosoluble molecule entrapped in a $\mathrm{A} / \mathrm{O} / \mathrm{A}$ multiple emulsion. Journal of Controlled Release, v. 45, p.1-13, 1997.

JIGAR, V.; ADARSH, S.; DHAVAL, R.; VIJAY, P. Development of stable multiple emulsion of atorvastatin. International Journal of Applied Biology and Pharmaceutical Technology, v. 2(2), p. $419-428,2011$.

KUMAR, R.; KUMAR, M.S.; MAHADEVAN, N. Multiple Emulsions: A Review. International Journal of Recent Advances in Pharmaceutical Research, v.2(1), p.9-19, 2012.

LOBATO-CALLEROS, C.; SOSA-PEREZ, A.; RODRIGUEZ-TAFOYA, J.; SANDOVALCASTILLA, O.; PEREZ-ALONSO, C.; VERNON-CARTER, E.J. Structural and textural characteristics of reduced-fat cheese-likeproducts made from W1/O/W2 emulsions and skim milk. LWT - Food Science and Technology, v.41, p.1847-1856, 2008.

MAHMOOD, T.; AKHTAR, N.; KHAN, B.A.; RASUL, A.; KHAN, H.M. Fabrication, physicochemical characterization and preliminary efficacy evaluation of a W/ON multiple 
emulsion loaded with $5 \%$ green tea extract. Brazilian Journal of Pharmaceutical Sciences, v. 49(2), p.341-349, 2013.

MAHMOOD, T.; AKHTAR, N.; MANICKAM, S. Interfacial film stabilized W/O/W nano multiple emulsions loaded with green tea and lotus extracts: systematic characterization of physicochemical properties and shelf-storage stability. Journal of Nanobiotechnology, v.12:20, p.1-8, 2014.

MATSUMOTO, S.; KITA, Y.; YONEZAWA, D. An attempt at preparing water-in-oil-in-water multiple-phase emulsions. Journal of Colloid and Interface Science, v. 57, $353-361$, 1976.

MORAIS, J.M. Desenvolvimento e avaliação do processo de obtenção de emulsões múltiplas $\mathrm{A} / \mathrm{O} / \mathrm{A}$ em etapa única empregando óleo de canola e tensoativo não iônico derivado do óleo de rícino. Tese de Doutorado em Ciências Farmacêuticas pela USP, Ribeirão Preto, 231 p., 2008.

OMOTOSHO, J. A.; LAW, T. K.; WHATELY, T. L.; FLORENCE, A. T. The stabilization of w/o/w emulsions by interfacial interaction between albumin and non ionic surfactants. Colloids and Surfaces, v.20, p.133-144, 1986.

OMOTOSHO, J. A. The effect of acacia, gelatin and polyvinylpyrrolidone on chloroquine transport from multiple W/O/W emulsions. International Journal of Pharmaceutics, v.62, p. 81-84. 1990.

PEREIRA, L.J.B.; GARCIA-ROJAS, E.E. Multiple emulsions: formation and application in microencapsulation of bioactive components. Ciência Rural, Santa Maria, v.45(1), p.155$162,2015$.

ROWE, R.C.; SHESKEY, P.J.; QUINN, M.E. Handbook of pharmaceutical excipients. Sixth edition, p. 917, 2009.

SCARIOTTO, M.C. Estudo da utilização da goma xantana como auxiliar no processo de floculação em tratamento de água para abastecimento. Trabalho de Conclusão de Curso 
de Graduação, do Curso Superior de Engenharia Ambiental da Coordenação de Engenharia Ambiental - COEAM - da Universidade Tecnológica Federal do Paraná UTFPR, 2013.

SCHMIDTS, T.; DOBLER, D.; NISSING, C.; RUNKEL, F. Influence of hydrophilic surfactants on the properties of multiple W/O/W emulsions. Journal of Colloid and Interface Science, v.338, p.184-192, 2009.

SCHMIDT, U.S; BERNEWITZ, R.; GUTHAUSEN, G.; SCHUCHMANN, H.P. Investigation and application of measurement techniques for the determination of the encapsulation efficiency of O/W/O multiple emulsions stabilized by hydrocolloid gelation. Colloids Surf. A: Physicochemical Eng. Aspects, v.475, p.55-61, 2015.

SCHMIDTS, T.; DOBLER, D.; SCHLUPP, P.; NISSING, H.; GARN, H.; RUNKEL, F. Development of multiple $\mathrm{W} / \mathrm{O} / \mathrm{W}$ emulsions as dermal carrier system for oligonucleotides: Effect of additives on emulsion stability. International Journal of Pharmaceutics, v.398, p.107-113. 2010.

SHICHIRI. M.; KAWAMORI, R.; YOSHUDA, M.; ETANI, N.; HOSHI, IZUMI, K.; SHIGETA, Y.; ABE, $H$.; Short term treatment of alloxan-diabetic rats with intrajejunal administration of water-in-oil-in-water insulin emulsions. Diabetes, v.24, p.971-976, 1975.

SIGWARD, E.; MIGNET, N.; RAT, P.; DUTOT, M.; MUHAMED, S.; GUIGNER, J-M.; SCHERMAN, D.; CRAUSTE-MANCIET, S. Formulation and cytotoxicity evaluation of new self-emulsifying multiple $\mathrm{W} / \mathrm{O} / \mathrm{W}$ nanoemulsions. International Journal of Nanomedicine, v.8, p.611-625, 2013.

TONELI, J.T.C.L.; MURR, F.E.X.; PARK, K.J. Estudo da reologia de polissacarídeos utilizados na indústria de alimentos. Revista Brasileira de Produtos Agroindustriais, Campina Grande, Especial, v.7, n.2, p.181-204, 2005. 